\title{
ORIGIN OF BINARY SYSTEMS
}

\author{
PETER BODENHEIMER \\ University of California Observatories \\ Lick Observatory \\ Board of Studies in Astronomy and Astrophysics \\ University of California, Santa Cruz, CA 95064, USA
}

\begin{abstract}
Recent observational studies of the properties of binary systems among young stars indicate that the majority of binaries are formed very early in the history of a star, perhaps during the protostellar collapse. Major observational facts to be explained include the overall binary frequency, the non-negligible occurrence of multiple systems, and the distributions of period, eccentricity, and mass ratio among the individual binaries. Theoretical calculations of the collapse of rotating protostars during the isothermal phase indicate instability to fragmentation into multiple systems. This process in general produces systems with periods greater than a few hundred years, although somewhat shorter periods are possible. Fragmentation during later, optically thick, phases of collapse tends to be suppressed by pressure effects. Therefore, major theoretical problems remain concerning the origin of close binaries. Fission of rapidly rotating stars, tidal capture, and three-body capture have been shown to be improbable mechanisms for formation of close binaries. Mechanisms currently under study include gravitational instabilities in disks, orbital interactions and disk-induced captures in fragmented multiple systems, hierarchical fragmentation, and orbital decay of long-period systems. Single stars, on the other hand, could result by escape from multiple systems or by the collapse of clouds of low angular momentum, coupled with angular momentum transport after disk formation.
\end{abstract}

Key words: stars: binaries - stars: formation - stars: rotation - stars: multiple systems

The determination of the mechanism of origin of binary systems presents numerous theoretical problems. This paper summarizes some relevant observational data, reviews a number of suggested formation processes, and concentrates on recent theoretical results, primarily $3-\mathrm{D}$ numerical hydrodynamical simulations. More detailed reviews have recently been presented by Bodenheimer, Ruzmaikina, \& Mathieu (1992), Pringle (1991), Boss (1991a), and Zinnecker (1990).

\section{Observations}

Although no direct observation of the formation of a binary system has been made, systems at very early stages have been identified (Wootten 1989; Sasselov \& Rucinski 1990). The formation process must explain the following observed properties of multiple systems (see above-mentioned reviews for more detailed references).

1) Approximately $70 \%$ of main-sequence solar-type stars have one or more companions (Abt 1983; Duquennoy \& Mayor 1991). Although surveys are not nearly as complete for other spectral classes on the main sequence, results indicate that these classes have very similar percentages (Abt 1983) with the possible exception of the M dwarfs, which appear to be deficient in short-period companions (Marcy $\&$ Benitz 1989). A survey of halo and thick-disk stars suggests that the frequency of spectroscopic binaries there is comparable to that of Population I stars (Torres 1991). Limited studies of pre-main-sequence stars (Mathieu, Walter, \& Myers 1989; Simon et al. 1991) suggest that the frequency is not noticeably different from that of main-sequence stars. 
2) The periods of main-sequence binaries form a smooth distribution in the range $0<\log \mathrm{P}$ (days) $<9$ with a single maximum at about 180 years (Duquennoy \& Mayor 1991).

3) The distribution of mass ratios among the longer-period binaries is consistent with random pairing from a normal initial mass function (Abt 1983), that is, the distribution rises toward smaller values of the mass ratio $q$. According to Duquennoy \& Mayor (1991) and Halbwachs (1987) there is no evidence that the $q$-distribution depends on binary period; that is, the same statement can be made about shortperiod systems. However Abt (1987) claims that the shorter-period binaries tend to have mass ratios closer to unity; further observations and analysis are necessary to clarify this point.

4) Binaries probably formed with a wide range of eccentricities. Field main-sequence systems with $\mathrm{P}<10$ days have circular orbits, probably as a result of post-formation tidal evolution. At longer periods, practically all orbits are eccentric (Duquennoy \& Mayor 1991).

5) Evidence has been found for disks associated with some young single stars and with some young binaries with a wide range of periods (Mathieu; this volume).

6) Most binaries formed during the star formation phase or during protostellar collapse, as indicated by the fact that binary stars are detected among pre-mainsequence stars with ages as young as $10^{5} \mathrm{yr}$ and that pre-main-sequence binary frequencies are no different from the corresponding main-sequence frequencies (Mathieu; this volume).

7) Evidence from observations of a few pre-main-sequence systems suggests that the components of a given binary are coeval, at least to within an age difference of $10^{6}$ yr (see Bodenheimer, Ruzmaikina, \& Mathieu 1992; Strom 1991).

8) Hierarchical multiple systems are common; they have a wide range of properties, but an example would be a primary with a close secondary with a period of 10 days and a wide companion with a period of 30 years. Most period ratios in such systems fall in the range 10 to $10^{4}$ (Fekel 1981).

\section{Basic formation mechanisms}

A number of different ideas have been put forward regarding the origin of binary systems. The original suggestion put forward by Laplace in 1796 (see Tassoul 1978) was that binaries formed from separate stellar nuclei during star formation; the nuclei then somehow came into orbit. The physics behind this picture was never fully explained, so in this review it will be assumed that the separate nuclei formed by fragmentation. Capture into orbit of two independently formed unbound stars in the presence of a dissipative process was suggested in 1867 by Stoney (see Aitken 1935). Fission, meaning the breakup by dynamical instability of a rapidly rotating object in hydrostatic equilibrium, is attributed to Kelvin and Tait in 1883 (see Tassoul 1978) and was strongly advocated by Jeans (1929).

Fragmentation, on the other hand, refers to the breakup of a rotating protostar during the hydrodynamical collapse phase. It was originally proposed by Hoyle (1953), who based the argument simply on the fact that an isothermal collapsing cloud, originally dynamically unstable on a large scale, becomes dynamically 
unstable on smaller and smaller scales because of the density increase during collapse. Later work has shown that rotation is a crucial element that stops the overall collapse of the cloud and allows individual fragments to separate out. The role of rotation in hierarchical fragmentation was investigated by Bodenheimer (1978). A variation of this process involves triggering of the fragmentation process by cloudcloud collisions (Pringle 1989), although Lattanzio \& Henriksen (1988) did not see formation of binaries as condensed objects in their parameter range of numerical simulations of isothermal cloud-cloud collisions. Pringle suggests that the highly elliptical binary orbits that are produced can then evolve to systems with smaller semi-major axes through disk interactions at periastron. By way of contrast, in "normal" fragmentation the individual clouds gradually evolve and develop dense cores which then spontaneously fragment after the onset of gravitational collapse. Finally, the calculations of Adams, Ruden, \& Shu (1989) indicate that the disks that form around young stars can, under the right conditions, become gravitationally unstable, in particular to the $m=1$ eccentric mode, and could possibly fragment. The following sections concentrate on the four basic processes of capture, fission, "normal" fragmentation, and disk instability.

\section{Capture}

Two independently formed stars can be captured into orbit if (1) a third body is present to take away the excess energy, if (2) the encounter is close enough so that tidal dissipation performs the same function, or if (3) a dissipative medium, such as residual gas in a young cluster, is present. Considering processes (1) and (2), expected capture rates in the galactic disk or in young dense clusters have been shown to be far too slow to explain the observed binary frequency (Hills 1976; Boss 1988; Hills \& Day 1976; Mansbach 1970). The presence of residual gas in the form of disks around young stars could change the picture. Larson (1990) showed that disk-induced captures in a very dense stellar environment such as the Orion Trapezium cluster occur at a significant rate (0.4 captures per star per $10^{6} \mathrm{yr}$ ). Clarke \& Pringle (1991a) showed that low-velocity encounters of stars with disks do result in capture, but previous high-velocity encounters may have removed the disks. They revise the capture rate found by Larson downward by an order of magnitude. Recent numerical calculations by Heller (1991) find an intermediate result: $\approx 0.05-0.1$ captures per star per $10^{6} \mathrm{yr}$ in Orion. Three-body captures produce wide separations, two-body tidal captures produce very close separations, and disk captures give separations comparable to the disk outer radius. The combination of such processes is unlikely to produce the observed smooth period distribution. The capture process also predicts (1) a wide range of eccentricities, as observed, (2) a wide range of mass ratios, essentially uncorrelated, as observed, (3) non-coevality of the components, for which there is no evidence except at the $10^{6} \mathrm{yr}$ level, and (4) non-alignment of the angular momentum in spin and orbit, for which there is evidence in some systems. However, even if capture is unlikely under normal circumstances, it could play a role in a slightly different situation. Suppose a molecular cloud core collapsed and fragmented, as illustrated in Figure 1 (see also Monaghan \& Lattanzio 1991). If the separations are only 0.01 parsec, the stellar density is 


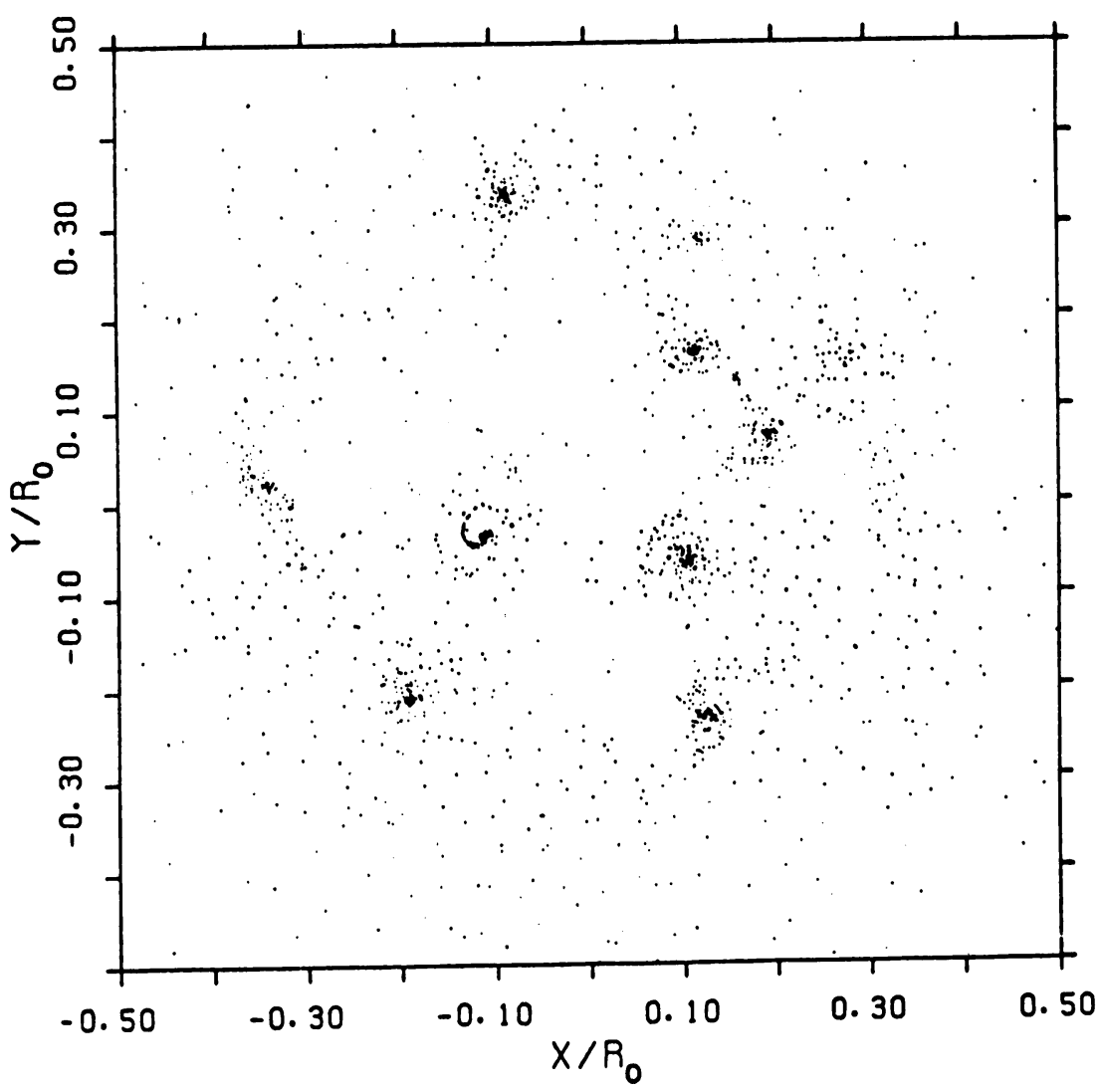

Fig. 1. Numerical SPH calculation of the fragmentation of a rotating, isothermal cloud (from Miyama, S, Hayashi, C., \& Narita, S. 1984, ApJ, 279, 621). Particle positions are projected into the equatorial $(x, y)$ plane, where the coordinates are normalized to the outer radius $R_{0}$.

considerably higher than it is in the Orion Trapezium cluster. The fragments will gravitationally interact, resulting in some ejections that perhaps will produce single stars, but also in a number of three-body or disk-induced captures, some into close orbits. The treatment of such systems as an N-body problem has been extensively investigated (see e. g. Anosova 1989). Clarke \& Pringle (1991b) have made numerical studies of the case of an initially co-orbiting (and highly unstable) three-body system, including disk dissipation, and have shown that the results are extremely sensitive to changes in the initial conditions and that a wide variety of orbital parameters and eccentricities is possible. 


\section{Fission}

As a star accumulates during protostar collapse, or as it contracts toward the main sequence with disk accretion, it tends to spin up, and the ratio $\beta$ of the rotational energy to the absolute value of the gravitational energy increases. When $\beta$ obtains a critical value, the star becomes unstable to non-axisymmetric perturbations. It has been hypothesized that breakup into orbiting subcondensations then occurs. Because only a small amount of angular momentum can be stored in a star, this mechanism would produce close binaries. The classical path to fission was based on the properties of uniform-density configurations (see Lyttleton 1953). The contracting sequence of Maclaurin spheroids becomes secularly unstable, in the presence of a dissipative mechanism, to deformation into triaxial objects (Jacobi ellipsoids), when $\beta=0.138$. Evolution was then envisaged to proceed along the sequence of contracting Jacobi ellipsoids until a point of dynamical instability to a pear-shaped mode was encountered at $\beta=.163$. Fission was thought to result, but analytical methods were not sufficient to demonstrate this outcome. An alternative path to fission that has been considered involves the continued axisymmetric contraction of the Maclaurin spheroids beyond the point of secular instability, until a point of dynamical instability to non-axisymmetric perturbations is reached at $\beta=0.274$. It has been shown that analogous points of instability exist along sequences of polytropes, that is, centrally condensed configurations (Ostriker \& Bodenheimer 1973).

The fission scenario has several major problems (Tassoul 1978). First, T Tauri stars are observed to rotate very slowly. Even if they were (uniformly) rotating at breakup, they would have insufficient angular momentum to reach a point of dynamical instability. Thus it would seem that fission could only occur during the period of buildup of the stellar core by accretion. Second, angular momentum considerations require the binary mass ratio to be $\sim 1: 10$, in disagreement with observations. Third, the required initial condition of high $\beta$ may never be reached. If the protostar had low enough angular momentum to form a very close binary, much of its mass will collapse directly onto the stellar core rather than onto a surrounding disk. Magnetic or convective effects in the ionized stellar core are likely to transport enough angular momentum out of the star into the disk to prevent the core from reaching the critical $\beta$, and a single star results (Ruzmaikina 1981a,b). Fourth, even if the critical $\beta$ were reached, several independent 3-dimensional numerical hydrodynamical calculations (Durisen et al. 1986) show that the result is not fission but transfer of angular momentum out from the core by spiral waves so that $\beta$ is reduced below the critical value.

Figure 2 shows a calculation by Williams \& Tohline (1988), starting from a polytrope of index 0.8 with $\beta=0.31$. The figure shows that on a time scale of about 10 central rotation periods the system develops spiral arms and that the eventual outcome is a more slowly rotating central object plus a ring. The same result occurs if the critical $\beta$ is approached (through accretion) from below (Boss 1989); this calculation uses a realistic equation of state and includes radiative transfer. It should be noted, however, that the initial condition in these numerical calculations, with the exception of that by Boss, differs from the "classical" approach to fission, 

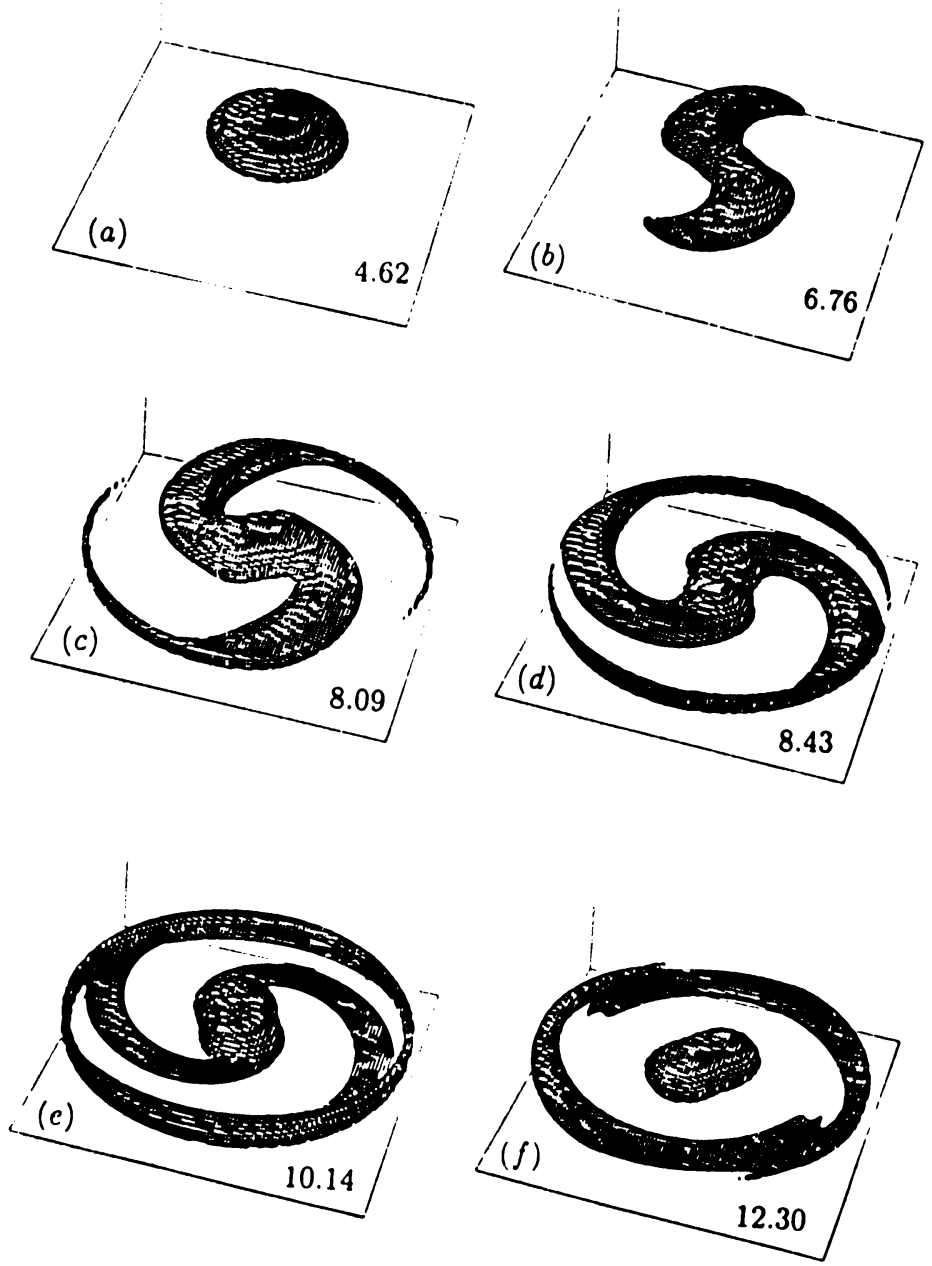

Fig. 2. The evolution in three dimensions of a rapidly rotating polytrope of index 0.8 with $\beta_{i}=0.31$ (from Williams, H. A., \& Tohline, J. E. 1988, ApJ, 334, 449). Equidensity surfaces at $10^{-3}$ of the central density are plotted at several different times, which are given in each frame in units of the initial central rotation period.

because it is analogous to starting near the point of dynamical (rather than secular) instability of the Maclaurin spheroid at $\beta=.274$ rather than at the dynamical instability of the Jacobi ellipsoid.

\section{Fragmentation}

Protostar collapse is divided into an early optically thin isothermal phase and a later optically thick adiabatic phase. Conditions for fragmentation are favorable during the isothermal phase. Three-dimensional hydrodynamical calculations for 
this phase generally start with a uniform-density sphere or spheroid, in uniform rotation, parametrized by initial values $\left(\alpha_{i}, \beta_{i}\right)$, which are, respectively, the thermal and rotational energies divided by the absolute value of the gravitational potential energy. A small initial density perturbation, either ordered or random, is introduced. According to Miyama, Hayashi, \& Narita (1984), fragmentation occurs if $\alpha_{i} \beta_{i}<$ 0.12 , as long as the cloud remains isothermal. Since $\beta_{i}$ has to be relatively large if rotational flattening (which is the necessary precursor to fragmentation) is to occur while the system is still isothermal, a long-period system is produced. However the typical interstellar cloud core has a power-law density profile $\rho \propto r^{-p}$, with $1<p<2$ (Myers et al. 1987). According to recent 3-D calculations, clouds with this density profile and uniform rotation do not fragment (Myhill \& Kaula 1991). However if this cloud is initially differentially rotating (Myhill \& Kaula 1991), or if it has a milder exponential-type density profile (Boss 1991b), it does fragment. Thus the criterion for fragmentation or for formation of a single star depends on the initial angular momentum distribution in the core of the molecular cloud.

During the adiabatic phase fragmentation tends to be suppressed because pressure effects cause perturbations to decay unless $\alpha_{i}$ is very small. For a gas of molecular hydrogen $(\gamma=1.4)$ it is found (Tohline 1981; Boss 1981) that the criterion for fragmentation becomes $\alpha_{i}<0.09 \beta_{i}^{0.2}$. Nevertheless, calculations starting in the isothermal phase have demonstrated that fragmentation can still occur in the central regions after they have already entered the adiabatic phase and that orbital separations as small as $\sim 1 \mathrm{AU}$ can result (Boss 1986). In the remainder of this section we discuss recent numerical calculations regarding fragmentation.

Boss (1991b) published results that apparently demonstrated numerically the occurrence of hierarchical fragmentation. An isothermal cloud with $\alpha_{i}=0.26, \beta_{i}$ $=0.16$ and a centrally condensed exponential density distribution started collapse with an $m=2$ azimuthal perturbation. After collapse to the point where rotational effects became important, a ring structure formed. The ring then started to fragment into a binary, and slightly later each fragment separated into two subfragments. However a true hierarchical fragmentation involves overall collapse of one of the original fragments to the point where rotational effects become important in it, in a manner analogous to the fragmentation of the original cloud. A typical fragment has a specific angular momentum in spin of only $5-10 \%$ that of the original cloud, so considerable collapse would be expected before subfragmention would occur. Such collapse is not followed in the Boss calculation. The scale of the orbit of the subfragments would be expected to be about 0.01 that of the original orbit; therefore the suborbit is unresolvable on the numerical grid that Boss used. Furthermore the subfragments were not shown to be in orbit about each other. Thus the Boss calculation does not represent true hierarchical fragmentation but rather a possible fragmentation mode of higher order than $m=2$.

An alternate mode of fragmentation was suggested by Zinnecker (1990), based on the observation that interstellar clouds often show an elongated, filamentary structure. He proposed that the filaments rotate about an axis perpendicular to that of the cylinder. After fragmentation of the cylinder (Bastien 1983) the fragments move towards each other along the cylindrical axis until they reach a kepler orbit. This idea has been explored numerically by Bonnell et al. (1991), who start 
with a cylindrical, isothermal cloud rotating end over end and follow the fragmentation with an SPH code containing approximately 2000 particles. The typical result is a binary system with separation $10^{3}-10^{4} \mathrm{AU}$ and high orbital eccentricity. Some circularization and orbital decay is expected because of dissipation induced by circumstellar matter. In one particular calculation the result was multiple fragmentation, consisting of a long-period system one of whose components was a binary with mass ratio $2: 1$ and the other a triple system with approximate mass ratios $3: 1: 1$.

A grid-based code in Cartesian coordinates has been developed by Myhill \& Kaula (1991), who consider the effects of initial differential rotation in a centrally condensed density distribution with $p=1$ or 2 , with a $10 \%$ or $50 \%$ initial perturbation. The angular momentum distribution is that of a uniformly rotating sphere of uniform density, under the hypothesis that magnetic braking was not completely effective in bringing the cloud to a state of uniform rotation. The result of a particular calculation with $p=1$, a $10 \%$ perturbation; $\alpha_{i}=0.17$, and $\beta_{i}=0.17$ is a binary with a separation of about $1000 \mathrm{AU}$, each component having a mass of about 0.2 of the original cloud mass. The spin angular momentum per unit mass in a condensation has been reduced by a factor 15 from that in the original cloud. The fragments have low enough thermal energy and rotational energy so that they are likely to fragment again, even though their further collapse will bring them into the adiabatic phase. Thus hierarchical fragmentation and the formation of short-period systems seems to be a definite possibility.

\section{Disk Fragmentation}

If a relatively slowly rotating protostar collapses through the adiabiatic phase without fragmenting, it will form a disk-like structure. Equilibrium keplerian disks around central stars have the possibility of fragmenting due to gravitational instabilities (Adams, Ruden, \& Shu 1989; Shu et al. 1990). If the Toomre $Q \approx \Omega c_{s} /(\pi G \sigma)>$ 1 , where $\Omega$ is the angular velocity, $c_{s}$ is the midplane sound speed, and $\sigma$ is the surface density, then a disk is locally stable against axisymmetric gravitational disturbances. However, it can be unstable to non-axisymmetric disturbances for $Q$ between 1 and $\approx 3$. The $m=1$ (one-armed spiral) mode may be crucial, according to the linear stability analysis of Adams et al. (1989). They find that if $Q=1$ at the outer edge, one can define a "maximum-mass solar nebula", corresponding to the point where the ratio of disk mass to total mass is 0.238 . Below this critical ratio, the disk is gravitationally stable to all modes. Above it, it is unstable with growth rates increasing with increasing mass and decreasing temperatures. The instability could result in spiral waves and resulting angular momentum transport by gravitational torques (Yang et al. 1991). In this case the end result would be a single star.

It is also possible that the instability could result in a binary companion, although a criterion for this event has not been established. A recent two-dimensional isothermal numerical SPH calculation by Benz (1991) shows incipient binary formation in a disk whose mass is equal to that of the central star and whose outer radius is about $30 \mathrm{AU}$. A $2.5 \%$ perturbation is applied initially, corresponding to 
a small offset of the central star from the center of mass. After a time of $100 \mathrm{yr}$ a companion with .03 of the disk mass forms on an elliptical orbit. This calculation must be considered preliminary in the sense that (1) without a perturbation of the right type binary formation does not occur, and (2) the assumed sharp outer edge of the disk is known to reinforce the instability, so that its role must be investigated more thoroughly.

\section{Summary}

Detailed numerical simulations, combined with observational data, are beginning to provide some clues regarding the dominant physical processes that govern binary formation. However, the range of input parameters in such calculations is wide, and the results often depend sensitively on them. The theory is not yet to the point where it can explain the observed distributions of binary period, mass ratio, and eccentricity. However, a few conclusions can already be reached.

First, fission has essentially been ruled out as a viable process for the formation of close binaries. Second, capture is unlikely to have produced many of the observed systems, although a few captures may have occurred in the cores of dense young clusters where circumstellar disks can act as the dissipation mechanism. Third, protostellar disks have been shown to be unstable, in the linear regime, to the growth of one-armed spiral modes as long as the disk mass fraction exceeds a critical value. It remains to be demonstrated, through non-linear numerical calculations, under what conditions this instability leads to binary formation. Fourth, fragmentation during the isothermal phase of protostar collapse has been demonstrated to produce binaries with long periods. Whether fragmentation occurs or not depends on initial conditions, in particular the angular momentum distribution.

The formation of close binaries is still a major issue, and it may require a combination of processes. At least three interesting suggestions should be further explored. First, at least some fragments that form during the isothermal collapse are suitable for subfragmentation as they evolve into the adiabatic phase. Thus, hierarchical fragmentation is likely to produce some close systems, and there is observational evidence to support this view. Second, an initial fragmentation stage during the isothermal collapse could produce a number of fragments in eccentric orbits. Gravitational interactions, disk dissipation, and captures in such a system could produce binary orbits with a wide range of periods and eccentricities. Third, the orbit of a long-period highly eccentric binary formed in a cloud-cloud collision or during the isothermal collapse could be reduced in semimajor axis through disk interactions near periastron. Thus the general mechanism of fragmentation could either directly or indirectly be responsible for the formation of a wide range of binary and multiple systems.

\section{Acknowledgements}

This work was supported in part by a special NASA Theory grant which provides funding for a joint Center for Star Formation Studies at NASA-Ames Research Center, University of California, Berkeley, and University of California, Santa Cruz. 
I thank Dr. Alan Boss, Dr. Elizabeth Myhill, Dr. Hugo Martel, Dr. Ian Bonnell, Dr. Jean-Pierre Arcoragi, and Dr. Willi Benz for providing me with information on their numerical calculations prior to publication. I also thank Dr. Robert Mathieu for his comments and the International Astronomical Union for a travel grant.

\section{References}

Abt, H. 1983, ARAA, 21, 343

Abt, H. 1987, ApJ, 317, 353

Adams, F. C., Ruden, S. P., \& Shu, F. H. 1989, ApJ, 347, 959

Aitken, R. G. 1935, The Binary Stars (New York: McGraw-Hill), 275

Anosova, J. P. 1989, in Highlights of Astronomy, vol. 8, ed. D. McNally (Dordrecht: Kluwer), 143

Bastien, P. 1983, A\&A, 119, 109

Benz, W. 1991, personal communication

Bodenheimer, P. 1978, ApJ, 224, 488

Bodenheimer, P., Ruzmaikina, T., \& Mathieu, R. 1992, in Protostars and Planets III, eds. E. Levy, J. Lunine, \& M. S. Matthews (Tucson: University of Arizona Press), in press.

Bonnell, I., Martel, H., Bastien, P, Arcoragi, J.-P., \& Benz, W. 1991, ApJ, 377, 553

Boss, A. P. 1981, ApJ, 250, 636

Boss, A. P. 1986, ApJS, 62, 519

Boss, A. P. 1988, Comments Ap, 12, 169

Boss, A. P. 1989, ApJ, 346, 336

Boss, A. P. 1991a, in Close Binaries, eds. J. Sahade, G. McCluskey, \& Y. Kondo (Dordrecht: Kluwer), in press.

Boss, A. P. 1991b, Nature, 351, 298

Clarke, C., and Pringle, J. E. 1991a, MNRAS, 249, 584

Clarke, C., and Pringle, J. E. 1991b, MNRAS, 249, 588

Duquennoy, A., \& Mayor, M. 1991, A\&A, 248, 485

Durisen, R. H., Gingold, R. A., Tohline, J. E., \& Boss, A. P. 1986, ApJ, 305, 281

Fekel, F. 1981, ApJ, 246, 879

Halbwachs, J.-L. 1987, A\&A, 183, 234

Heller, C. 1991, Ph. D. dissertation, Yale University

Hills, J. G. 1976, MNRAS, 175, 1P.

Hills, J. G., and Day, C. A. 1976, ApL, 17, 87

Hoyle, F. 1953, ApJ, 118, 513

Jeans, J. H. 1929, Astronomy and Cosmogony, 2nd ed. (Cambridge: Cambridge University Press)

Larson, R. B. 1990, in Physical Processes in Fragmentation and Star Formation, eds. R. Capuzzo-Dolcetta, C. Chiosi, \& A. DiFazio (Dordrecht: Kluwer), 389

Lattanzio, J. C., \& Henriksen, R. N. 1988, MNRAS, 232, 565

Lyttleton, R. A. 1953, The Stability of Liquid Rotating Masses (Cambridge: Cambridge University Press)

Mansbach, P. 1970, ApJ, 160, 135 
Marcy, G. W., \& Benitz, K. J. 1989, ApJ, 344, 441

Mathieu, R., Walter, F., \& Myers, P. 1989, AJ, 98, 987

Miyama, S., Hayashi, C., \& Narita, S. 1984, ApJ, 279, 621

Monaghan, J. J., \& Lattanzio, J. C. 1991, ApJ, 375, 177

Myers, P. C., Fuller, G. A., Mathieu, R. D., Beichman, C. A., Benson, P. J., Schild,

R. E., \& Emerson, J. P, 1987, ApJ, 319, 340

Myhill, E. A., and Kaula, W. M. 1991, ApJ, in press

Ostriker, J. P., \& Bodenheimer, P. 1973, ApJ, 180, 171

Pringle, J. E. 1989, MNRAS, 239, 361

Pringle, J. E. 1991, in Physics of Star Formation and Early Stellar Evolution, eds. C. Lada \& N. Kylafis (Dordrecht: Kluwer), 437

Ruzmaikina, T. V. 1981a, Adv. Space Res., Vol 1, No 7, 49

Ruzmaikina, T. V. 1981b, Pisma Astr. J. U. S. S. R., 7, 188

Sasselov, D., \& Rucinski, S. 1990, ApJ, 351, 578

Shu, F. H., Tremaine, S., Adams, F. C., \& Ruden, S. P. 1990, ApJ, 358, 495

Simon, M., Chen, W. P., Howell, R. R., Benson, J. A., \& Slowik, D. 1991, ApJ, in press

Strom, K. 1991, in preparation

Tassoul, J.-L. 1978, Theory of Rotating Stars (Princeton: Princeton University Press)

Tohline, J. E. 1981, ApJ, 248, 717

Torres, G. 1991, Ph. D. dissertation, Córdoba University, Argentina

Williams, H. A., and Tohline, J. E. 1988, ApJ, 334, 449

Wootten, A. 1989, ApJ, 337, 858

Yang, S., Durisen, R. H., Cohl, H., Imamura, J. N., \& Toman, J. 1991, Icarus, 91, 14

Zinnecker, H. 1990, in Low Mass Star Formation and Pre-Main-Sequence Objects, ed. B. Reipurth (Garching: European Southern Observatory), 447 